\title{
Wide Area Digital Dermoscopy for Congenital Melanocytic Nevi: Report of 3 Cases
}

\author{
Guilherme A. Gadens, ${ }^{1}$ Gerson Dellatorre ${ }^{1}$
}

1 Department of Dermatology \& Cutaneous Oncology, Hospital Santa Casa de Curitiba, Brazil

Key words: melanocytic nevi, image enhancement, dermoscopy, pigmented nevus

Citation: Gadens GA, Dellatorre G. Wide area digital dermoscopy for congenital melanocytic nevi: report of 3 cases. Dermatol Pract Concept. 2020;10(1):e2020023. DOI: https://doi.org/10.5826/dpc.1001a23

Accepted: October 23, 2019; Published: December 31, 2019

Copyright: $@ 2019$ Gadens and Dellatorre. This is an open-access article distributed under the terms of the Creative Commons Attribution License, which permits unrestricted use, distribution, and reproduction in any medium, provided the original author and source are credited.

Funding: None.

Competing interests: The authors have no conflicts of interest to disclose.

Authorship: Both authors have contributed significantly to this publication.

Corresponding author: Gerson Dellatorre, MD, Alameda Prudente de Moraes, 1250, Curitiba, Paraná, Brazil, 80430-220. Email: dellatorre@gmail.com

\section{Introduction}

Digital dermoscopy has an essential role in diagnosing a variety of skin lesions once it allows their follow-up over time. The diameter of the dermatoscope lenses limits the applicability of this tool in the diagnosis of large lesions. We present a case series of 3 congenital melanocytic nevi (CMN) documented by the wide area digital dermoscopy (WADD) technique, which allows a dermoscopic record of the entire lesion area.

As previously described [1], the WADD technique consists of merging multiple and overlapped separate dermoscopic images by using the "Photomerge" feature of Adobe Photoshop CC software (Adobe Systems Incorporated, San Jose, CA, USA, v19.1.6). The final processed image comprises the full lesion at a high resolution. Dermoscopic definition of every single separate image is completely preserved, allowing navigation throughout the lesion with pan and zoom capabilities.

\section{Case Presentation}

Our case series comprised 3 patients with CMN. Figure 1 shows CMN located on the plantar area in a 23 -year-old patient. In Figure 2 the CMN are located on the right malar area in a 20-month-old patient. Figure 3 shows CMN located on the left gluteal region in a 37 -year-old patient.

\section{Conclusions}

Clinical and dermoscopic follow-up studies of CMN are essential for early detection of malignant transformation. WADD technique may improve follow-up once it allows the comparison of an entire large area. Applicability of this technique extends beyond $\mathrm{CMN}$, as it can be useful in any situation involving digital dermoscopy of large lesions. As a limitation, scanning of ultra-large lesions can be challenging, demanding time to obtain the required image overlapping correctly. In the future, specific and user-friendly software may contribute to facilitating the process of image acquiring and processing.

\section{Reference}

1. Dellatorre G, Gadens GA. Wide area digital dermoscopy. J Am Acad Dermatol. 2019;80(6):e153. 


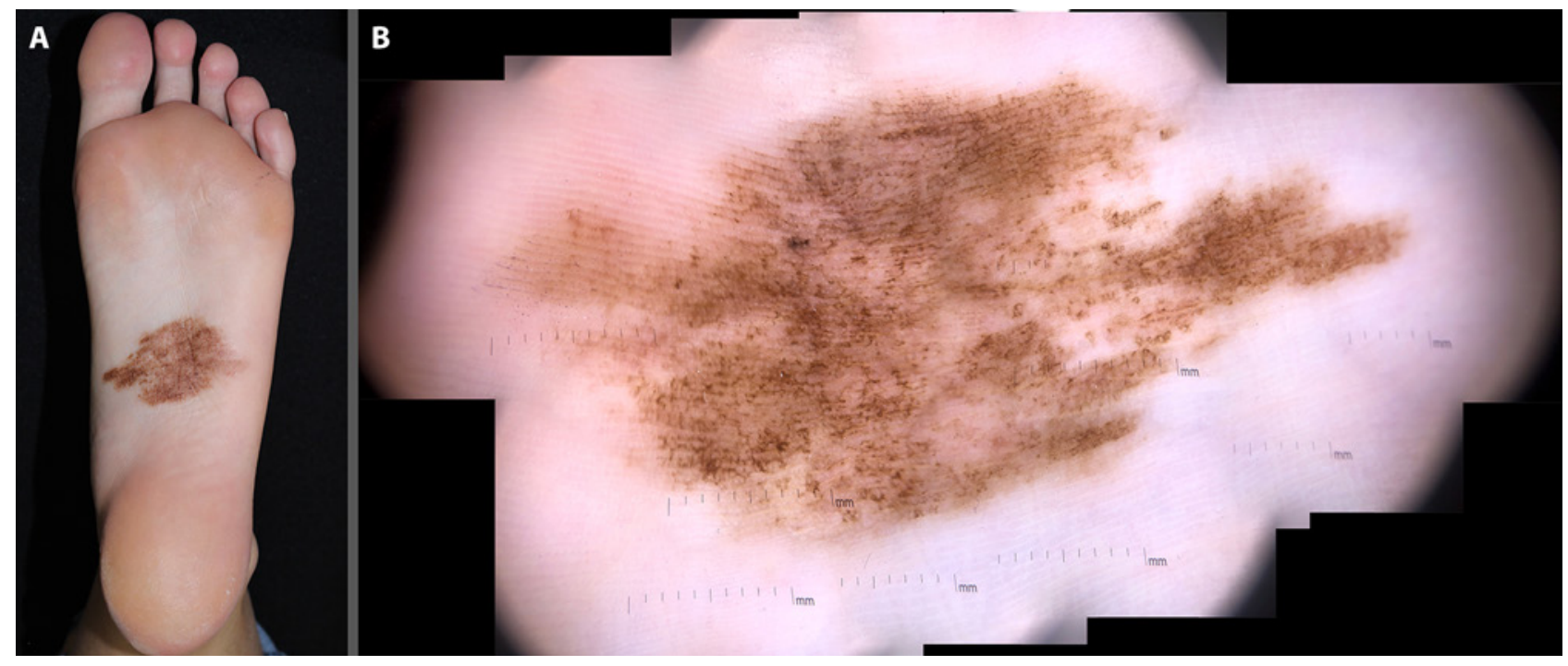

Figure 1. (A) Congenital melanocytic nevi measuring $6.3 \times 3.4 \mathrm{~cm}$ located on the plantar area in a 23 -year-old patient. (B) Wide area digital dermoscopy obtained from merging of 20 separate dermoscopic images (DermLite DL4 3GEN Inc., San Juan Capistrano, CA, USA; attached to a smartphone; polarized, magnification $\times 10)$.

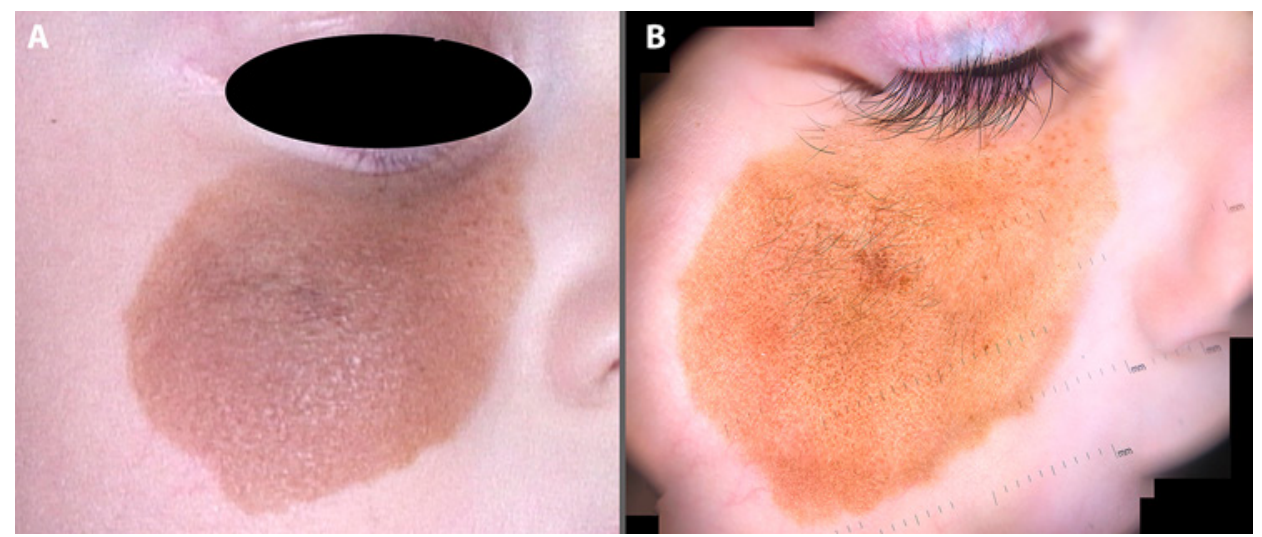

Figure 2. (A) Congenital melanocytic nevi measuring $4.9 \times 2.9 \mathrm{~cm}$ located on the right malar area in a 20-month-old patient. (B) Wide area digital dermoscopy obtained after merging of 17 dermoscopic images.

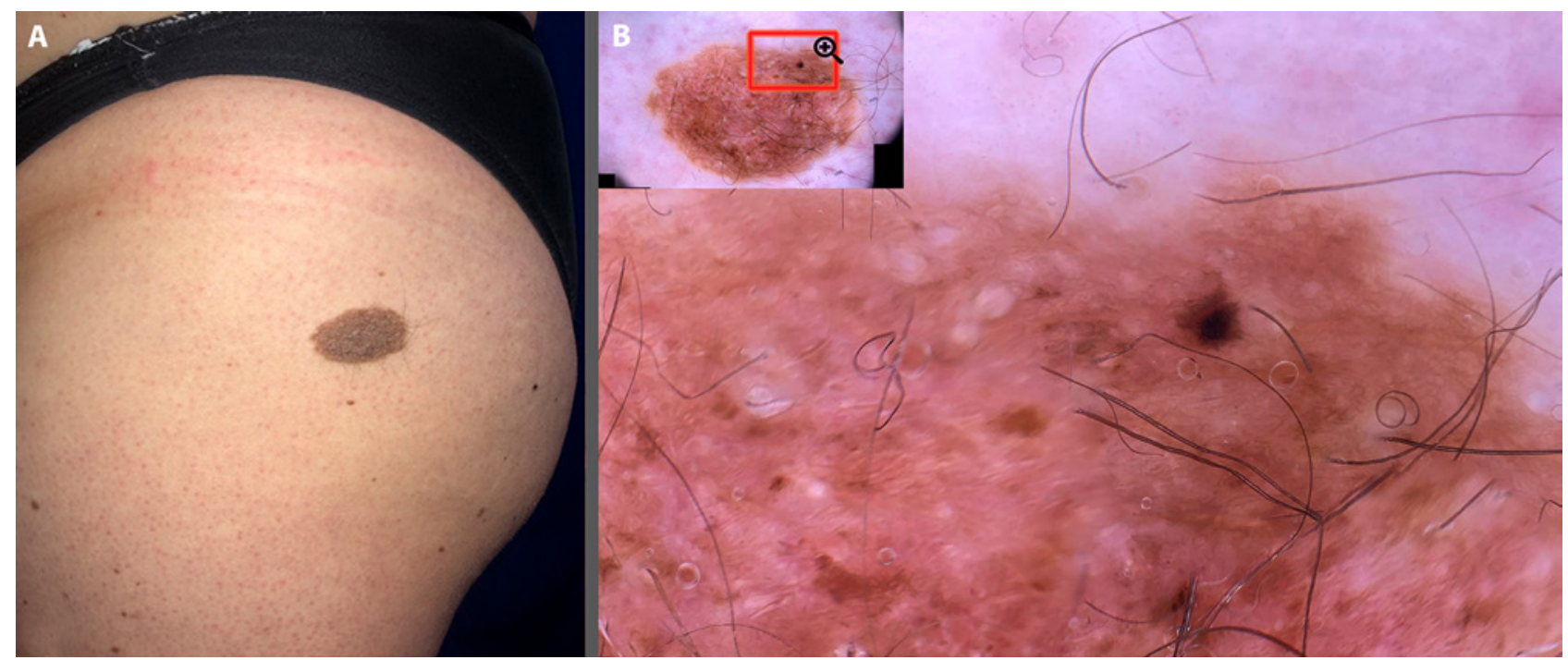

Figure 3. (A) Congenital melanocytic nevi measuring $3.3 \times 2.2 \mathrm{~cm}$ located on the left gluteal region in a 37-year-old patient. (B) Detail of zoom capability, preserving dermoscopic definition, of final wide area digital dermoscopy image obtained from 14 separate dermoscopic images. 